\title{
THE METRICAL ASPECT OF THE LINE-SPHERE TRANSFORMATION*
}

\author{
BY
}

\section{JULIAN LOWELL COOLIDGE}

The line-sphere transformation of Sophus Lie, first described in his remarkable article, "Ueber Complexe, insbesondere Linien und Kugelcomplexe, mit Anwendung auf die Theorie partieller Differentialgleichungen" $\dagger$ derives its interest from two causes. Firstly, lines and spheres are the simplest fourdimensional geometrical manifolds with which we have to deal; it is interesting to establish connections between them. Secondly, intersecting lines correspond to tangent spheres. The consequence usually drawn from this is that the linesphere transformation falls under the general type of contact transformation, and has the beautiful property of carrying asymptotic lines over into lines of curvature. These facts are of capital importance when the subject is approached from the point of view of Pfaff equations. But we may adopt a different point of view, and then the question naturally arises: "Can not the correspondence of intersecting lines with tangent spheres be made to appear as a special case of some more general relation connecting the distances of lines with the angles of intersection of spheres?" It is the object of the present paper to answer this question affirmatively, to develop as far as possible the relations between the metrical properties of line and sphere systems, and to bring the two into the closest possible connection. $\ddagger$

Before proceeding to our task, two words of caution are necessary. First, as the angles between spheres appear most naturally through their trigonometric functions, the corresponding line-functions are found most elegantly in non-euclidean space, where distances usually appear in trigonometric form. Secondly, the group of contact transformations that carries lines into lines and keeps distances invariant depends upon six parameters, the group that leaves the angles of spheres intact is a ten parameter group, isomorphic with the quinary orthogonal one. We must, therefore, introduce some seemingly arbitrary restrictions, i. e.,

* Presented to the Society December 29, 1910.

$\dagger$ Mathematische Annalen, vol. 5 (1872).

† Since this paper was written my attention has been called to one written in a similar spirit by SEGRE, Sulle geometrie metriche dei complessi e delle sfere. Atti della R. Accademia delle Scienze di Torino, vol. 19 (1884). It is to be noted that Share deals with nonoriented spheres, and euclidean line space, so that he is only able to establish a certain similarity in the formulæ, not an identity as in the present case. 
assume the invariance of certain additional relations in our sphere system, in order to scale our group down to the proper size.

\section{$\S 1$. The Line Space $\Sigma$.}

In a three-dimensional space $\sum$ let a point have four homogeneous coördinates *

$$
\xi_{0}: \xi_{1}: \xi_{2}: \xi_{3} \text {. }
$$

The system of measurement shall be of the elliptic type, the equation of the Absolute

$$
\sum_{i}^{0} \xi_{i}^{2}=0
$$

The unit of measure shall be so chosen that the distance $d$ of $(\xi)$ and $(\eta)$ is given by

$$
\cos d=\frac{\sum_{i}^{0} \ldots_{i}^{3} \xi_{i} \eta_{i}}{\sqrt{\sum_{i}^{0} \dddot{1}^{3} \xi_{i}^{2}} \sqrt{\sum_{i}^{0} \dddot{M}^{3} \eta_{i}^{2}}} .
$$

In like manner the angle $\theta$ of the two planes

will have as cosine

$$
\sum_{i}^{0} u_{i}^{3} \xi_{i}=0, \quad \sum_{i}^{0} v_{i}^{3} \xi_{i}=0
$$

$$
\cos \theta=\frac{\sum_{i}^{0 \ldots 3} u_{i} v_{i}}{\sqrt{\sum_{i}^{0} \dddot{M}^{3} u_{i}^{2}} \sqrt{\sum_{i}^{0} \dddot{M}^{3} v_{i}^{2}}} \cdot
$$

A line $(p)$ shall be given by its six Plücker coördinates

$$
p_{01}: p_{02}: p_{03}: p_{23}: p_{31}: p_{12}, \quad \rho p_{i j}=\xi_{i} \eta_{j}-\xi_{j} \eta_{i}=u_{k} v_{l}-u_{l} v_{k},
$$

where the numbers $k, l$ are complementary to $i, j$ in the system $0,1,2,3$. The polar of $(p)$ with regard to the Absolute, or as we shall say, its absolute polar will have the coördinates

$$
p_{i j}^{\prime}=\sigma p_{k l}
$$

The condition for the intersection of two lines $(p)$ and $(q)$ will be

$$
\sum_{i j k l}^{0 \cdots 3} p_{i j} q_{k l}=0
$$

Each will intersect the polar of the other with regard to the Absolute if

$$
\sum_{i j}^{0} p_{i j} q_{i j}=0 \text {. }
$$

* The developments of the present section will be found at greater length in chapters IX, $X$ of the author's Elements of Non-euclidean Geometry, Oxford, 1909. 
Both expressions will vanish if they intersect orthogonally. A line will touch the Absolute if

The expression

$$
\sum_{i j}^{0 \cdots 3} p_{i j}^{2}=0
$$

$$
J=\frac{\sum_{i j k l}^{0 \ldots 3} p_{i j} q_{k l}}{\sum_{i j}^{3} p_{i j} q_{i j}}
$$

will be an absolute invariant under the group of all congruent transformations of space. What will be its meaning?

Two lines in elliptic space have two distances, the distances of their intersections with either of the two lines that cut them both orthogonally. They have also two angles, the angles of the pairs of planes determined by the given lines and by each of these perpendiculars. Let one perpendicular cut the two lines in $(\xi)$ and $\left(\xi^{\prime}\right)$, the other cutting them in $(\eta)$ and $\left(\eta^{\prime}\right)$. We have for the product of the cosines of the distances $d_{1}$ and $d_{2}$ of the lines

$$
\cos d_{1} \cos d_{2}=\frac{\sum_{i}^{0} \xi_{i} \xi_{i}^{\prime} \sum_{i}^{3} \eta_{i} \eta_{i}^{\prime}}{\sqrt{\sum_{i}^{0} \xi_{i}^{3}} V^{10} \dddot{\sum}_{i}^{3} \eta_{i}^{2} \sqrt{\sum_{i}^{0} \dddot{m}^{3} \xi_{i}^{\prime 2}} \sqrt{\sum_{i}^{0} \dddot{m}_{i}^{\prime 2}}} .
$$

Now, since the pair $(\xi)\left(\xi^{\prime}\right)$ are orthogonal to $(\eta)\left(\eta^{\prime}\right)$, i. e., each point lies in the absolute polar plane of each point of the other pair: therefore

$$
\begin{aligned}
& \sum_{i}^{0} \dddot{\aleph}^{3} \xi_{i} \eta_{i}=\sum_{i}^{0} \dddot{\sum}^{3} \xi_{i} \eta_{i}^{\prime}=\sum_{i}^{0} \dddot{\aleph}^{3} \xi_{i}^{\prime} \eta_{i}=\sum_{i}^{0} \dddot{\aleph}^{3} \eta_{i}^{\prime} \eta_{i}^{\prime}=0, \\
& \sum_{i j}^{0} \dddot{\sum}^{3} p_{i j} p_{i j}^{\prime}=\sum_{i}^{0} \dddot{\sum}^{3} \xi_{i} \xi_{i}^{\prime} \sum_{i}^{3} \eta_{i} \eta_{i}^{\prime}, \\
& \sum_{i j}^{0} \dddot{\sum}^{3} p_{i j}^{2}=\sum_{i}^{0} \dddot{\aleph}^{3} \xi_{i}^{2} \varlimsup_{i}^{3} \eta_{i}^{2} \\
& \cos d_{1} \cos d_{2}=\frac{\sum_{i j}^{0} p_{i j} p_{i j}^{\prime}}{\sqrt{\sum_{i j}^{0} p_{i j}^{2} \sqrt{\sum_{i j}^{0} \dddot{M}^{3} p_{i j}^{\prime 2}}}} .
\end{aligned}
$$

For the product of the sines of the distances we have likewise

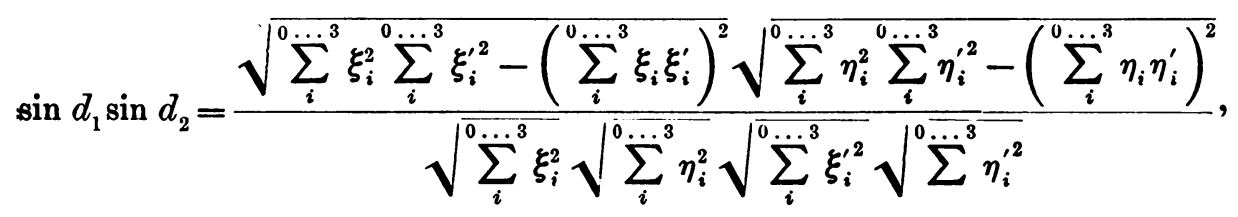


$\left[\sum_{i j k l}^{0} \dddot{3}^{3}\left(p_{i j} p_{k l}^{\prime}\right)\right]^{2}=\left|\xi \eta \xi^{\prime} \eta^{\prime}\right|^{2}$

therefore,

$$
=\left[\sum_{i}^{0} \dddot{\xi}_{i}^{3} \xi_{i}^{0} \dddot{\sum}_{i}^{3} \xi_{i}^{\prime 2}-\left(\dddot{\sum}_{i}^{0} \xi_{i} \xi_{i}^{\prime}\right)^{2}\right]\left[\sum_{i}^{0} \dddot{\eta}_{i}^{2} \dddot{\sum}_{i}^{3}{\eta_{i}^{\prime 2}}^{2}-\left(\sum_{i}^{0} \dddot{\eta}_{i} \eta_{i}^{\prime}\right)^{2}\right]
$$

$$
J \equiv \frac{\sin d_{1} \sin d_{2}}{\cos d_{1} \cos d_{2}}=\tan d_{1} \tan d_{2}
$$

We may say, in particular: for oblique intersection, $J$ vanishes; for the intersection of each line with the absolute polar of the other, $J$ is infinite; for orthogonal intersection, $J$ is indeterminate; when the two distances of the lines are complementary, $J$ equals unity.

The angles of two planes are equal to the distances of their absolute polar lines. But our expression (9) is unaltered if each Plücker coördinate be replaced by its complementary. Hence also

$$
J=\tan \theta_{1} \tan \theta_{2}
$$

The first interpretation is, however, the one which we shall follow in the present paper.

Two lines shall be said to be parallel* when they intersect in a point of the Absolute, they are pseudo-parallel $\dagger$ if they lie in a plane tangent to the Absolute. The necessary and sufficient condition for the existence of one of these relations is that the lines should intersect, and that there should be only one line of their pencil tangent to the Absolute,

$$
\sum_{i j k l}^{0} \dddot{3}^{3} p_{i j} q_{k l}=\sum_{i j}^{0}{\dddot{m}^{3}}_{i j}^{2} \sum_{i j}^{3} q_{i j}^{2}-\left({ }^{0} \dddot{M}^{3} p_{i j} q_{i j}\right)^{2}=0
$$

Two lines shall be said to be paratactic $\ddagger$ if they intersect the same two generators of the same system of the Absolute. What are the Plücker coördinates of such a generator?

The coördinates of a point of the Absolute may be expressed thus,

$\xi_{0}: i \xi_{1}: \xi_{2}: i \xi_{3}=\left(\lambda_{1} \mu_{1}-\lambda_{2} \mu_{2}\right):\left(\lambda_{1} \mu_{1}+\lambda_{2} \mu_{2}\right):\left(\lambda_{1} \mu_{2}+\lambda_{2} \mu_{1}\right):\left(\lambda_{1} \mu_{2}-\lambda_{2} \mu_{1}\right)$.

The Plücker coördinates of the generators of one set, which, for definiteness

* This is Lobachevski's form of parallelism.

†This word seems to have been coined by STUDY, Veber Nicht-Euklidische und Linien Geometrie, Jahresbericht der Dentschen Mathematiker-Vereinigung, vol. 15 (1906), pp. 489-490.

† The more usual name is Clifford Parallel. For "parataxy" see Srudy, loc. cit., or another article of same title, ibid., vol. 8 (1902), p. 317. 
we shall call the left, will be

$$
l_{01}=l_{23}=2 \lambda_{1} \lambda_{2}, \quad l_{02}=l_{31}=i\left(\lambda_{1}^{2}+\lambda_{2}^{2}\right), \quad l_{03}=l_{12}=\left(\lambda_{1}^{2}-\lambda_{2}^{2}\right)
$$

the right generators may similarly be written

$$
r_{01}=-r_{23}=2 \mu_{1} \mu_{2}, \quad r_{02}=-r_{31}=i\left(\mu_{1}^{2}+\mu_{2}^{2}\right), \quad r_{03}=-r_{12}=-\left(\mu_{1}^{2}-\mu_{2}^{2}\right) \text {. }
$$

Two lines $(p)$ and $\left(p^{\prime}\right)$ will be left paratactic if

$$
\begin{aligned}
& p_{01}+p_{23}=\rho\left(p_{01}^{\prime}+p_{23}^{\prime}\right), \\
& p_{02}+p_{31}=\rho\left(p_{02}^{\prime}+p_{31}^{\prime}\right), \\
& p_{03}+p_{12}=\rho\left(p_{03}^{\prime}+p_{12}^{\prime}\right) .
\end{aligned}
$$

For right parataxy we have similarly

$$
\begin{aligned}
& p_{01}-p_{23}=\sigma\left(p_{01}^{\prime}-p_{23}^{\prime}\right), \\
& p_{02}-p_{31}=\sigma\left(p_{02}^{\prime}-p_{31}^{\prime}\right), \\
& p_{03}-p_{12}=\sigma\left(p_{03}^{\prime}-p_{12}^{\prime}\right) .
\end{aligned}
$$

For parataxy, right or left, we have

$$
\left[\sum_{i j k l}^{0} p_{i j}^{3} p_{k l}^{\prime} \pm \sum_{i j}^{0} \dddot{m}_{i j} p_{i j}^{\prime}\right]^{2}=\left[\sum_{i j}^{0} \dddot{m}^{3} p_{i j}^{2}\right]\left[\sum_{i j}^{0} p_{i j}^{\prime 2}\right]
$$

and from this equation it will appear that when two lines are paratactic, their distances and angles are equal. Conversely, when the distances are equal

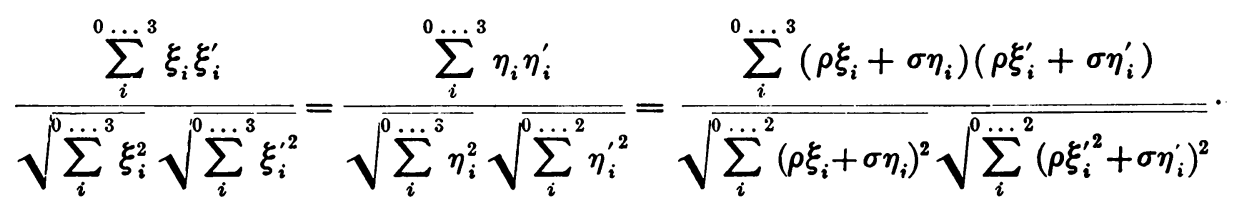

There are thus $\infty$ pairs of points whose distances are the distances of the lines, the lines have $\infty^{1}$ common perpendiculars, and so are paratactic.

It is better for our present purpose not to use Plücker coördinates, but the Klein coördinates related to them as follows :

$$
\begin{array}{ll}
p_{01}=\rho\left(x_{0}+i x_{3}\right), & p_{23}=\rho\left(x_{0}-i x_{3}\right), \\
p_{01}=\rho\left(x_{1}+i x_{4}\right), & p_{31}=\rho\left(x_{1}-i x_{4}\right), \\
p_{03}=\rho\left(x_{2}+i x_{3}\right), & p_{12}=\rho\left(x_{2}-i x_{5}\right) .
\end{array}
$$

Here $i$ is a square root of -1 whose value is supposed to be well determined. 
We have then

$$
\begin{array}{ll}
x_{0}=\sigma\left(p_{01}+p_{23}\right), & x_{3}=\sigma i\left(p_{01}-p_{23}\right), \\
x_{1}=\sigma\left(p_{02}+p_{31}\right), & x_{4}=\sigma i\left(p_{02}-p_{31}\right), \\
x_{2}=\sigma\left(p_{03}+p_{12}\right), & x_{5}=\sigma i\left(p_{03}-p_{12}\right) .
\end{array}
$$

The coördinates $x_{0}: x_{1}: x_{2}$ are proportional to the three non-vanishing Plücker coördinates of the lines through $(1,0,0,0)$ left paratactic to the given line, while $x_{3}: x_{4}: x_{5}$ are thenon-vanishing coördinates of the right paratactic through the same point. We get the Absolute polar of a given line by changing the signs of the first three, or last three Klein coördinates; it will be more convenient to adopt the latter alternative. Let us write our general scheme of formulæ in the new coördinates. Intersection of two lines :

$$
x_{0} y_{0}+x_{1} y_{1}+x_{2} y_{2}+x_{3} y_{3}+x_{4} y_{4}+x_{5} y_{5} \equiv(x y)=0, \quad(x x) \equiv(y y) \equiv 0
$$

Each intersects the absolute polar of the other if

$$
x_{0} y_{0}+x_{1} y_{1}+x_{2} y_{2}-x_{3} y_{3}-x_{4} y_{4}-x_{5} y_{5} \equiv(x \mid y)=0
$$

Orthogonal intersection :

$$
J=\frac{(x y)}{(x \mid y)}
$$

$$
(x y)=(x \mid y)=0 \text {. }
$$

Parallelism or pseudo-parallelism :

Right parataxy :

$$
(x y)=(x \mid x)(y \mid y)-(x \mid y)^{2}=0 .
$$

Left parataxy :

$$
x_{3}=y_{3}, \quad x_{4}=y_{4}, \quad x_{5}=y_{5} \text {. }
$$

Parataxy in general :

$$
x_{0}=y_{0}, \quad x_{1}=y_{1}, \quad x_{2}=y_{2} .
$$

$$
[(x y) \pm(x \mid y)]^{2}=(x \mid x)^{2}(y \mid y)^{2}
$$

Left generators of Absolute :

Right generators of Absolute :

$$
x_{3}=x_{4}=x_{5}=0
$$

$$
x_{0}=x_{1}=x_{2}=0 \text {. }
$$

We shall refer to these formulæ as set (iे0).

\section{§2. Sphere Space $S$.}

In a three-dimensional euclidean space $S$ let a point have the homogeneous rectangular cartesian coördinates $x: y: z: t$. The equation of any sphere may be written

$$
i x_{1}\left(x^{2}+y^{2}+z^{2}+t^{2}\right)+x_{2}\left(x^{2}+y^{2}+z^{2}-t^{2}\right)+x_{3}(2 x)+x_{4}(2 y)+{ }_{0}(2 z)=0 .
$$


The radius will be

$$
r=\frac{\sqrt{\sum_{i}^{1 \ldots 5} x_{i}^{2}}}{i x_{1}+x_{2}} .
$$

The centre will have coördinates

$$
x: y: z: t=-x_{3}:-x_{4}:-x_{5}: i x_{1}+x_{2} \text {. }
$$

Let us next assume that the radius may be either positive or negative, and say that a sphere has been oriented when the sign of the radius has been determined. A plane shall be said to be oriented if the normals have an assigned sense. Hereafter the words sphere and plane shall be taken to mean oriented sphere and oriented plane. Let us write

$$
x_{0}^{2}=-\left(x_{1}^{2}+x_{2}^{2}+x_{3}^{2}+x_{4}^{2}+x_{5}^{2}\right) \text {, }
$$

so that

$$
r=\frac{i x_{0}}{i x_{1}+x_{2}}
$$

We see that an oriented sphere has six homogeneous coördinates

$$
x_{0}: x_{1}: x_{2}: x_{3}: x_{4}: x_{5}, \quad(x x) \equiv 0 .
$$

The angle of intersection $\theta$ of two non-oriented spheres being given by

$$
\cos \theta=\frac{\sum_{i}^{1 \ldots 5} x_{i} y_{i}}{\sqrt{\sum_{i}^{1 \ldots 5} x_{i}^{2}} \sqrt{\sum_{i}^{1 \ldots 5} y_{i}^{2}}},
$$

we shall define as the angle of intersection of two oriented spheres $(x)$ and $(y)$

$$
\theta=\cos ^{-1} \frac{\sum_{i}^{1 \ldots 5} x_{i} y_{i}}{-x_{0} y_{0}}
$$

They shall be said to be tangent if $\cos ^{2} \theta=1$ and to touch if $\cos \theta=1$. Two tangent spheres whose radii have like signs will touch if their contact be internal.

If one of the oriented spheres be a plane, (25) will give the angle between the directed normal and the internal direction of the radius, if the latter be positive, otherwise the external direction. If both spheres be planes we have the angle of their directed normals. Let us replace (25) by the more convenient

$$
\sin ^{2} \frac{1}{2} \theta=\frac{(x y)}{2 x_{0} y_{0}}
$$

Trans. Am. Math. Soc. 4 
The condition that two spheres should touch is

$$
(x y)=0 \text {. }
$$

If we reverse the signs of $x_{3}: x_{4}: x_{5}$ we get the reflection of the given oriented sphere in the origin. We shall understand by reflection of a sphere the operation which replaces each point by its reflection and does not alter the orientation of the radius. If $\theta^{\prime}$ be the angle which each of our spheres makes with the reflection of the other in the origin, we get the fundamental formulæ

$$
\sin ^{2} \frac{1}{2} \theta^{\prime}=\frac{(x \mid y)}{x_{0} y_{0}}, \quad J=\frac{(x y)}{(x \mid y)}=\frac{\sin ^{2} \frac{1}{2} \theta}{\sin ^{2} \frac{1}{2} \theta^{\prime}} .
$$

This last equation has a meaning even in the case of null spheres. If two proper spheres have radii $r, r_{1}$, while the distance of their centres is $d$ and their angle of intersection is $\theta$,

$$
d^{2}=r^{2}+r_{1}^{2}-2 r r_{1} \cos \theta, \quad d^{2}-r^{2}=-\frac{x_{0}^{2}}{\left(i x_{1}+x_{2}\right)^{2}}+\frac{2 \sum_{i}^{1} \dddot{m}^{5} x_{i} y_{i}}{\left(i x_{0}+x_{1}\right)\left(i y_{0}+y_{1}\right)} .
$$

When the sphere $(x)$ becomes null with a finite centre, the expression on the left is the power of that centre with regard to the other sphere; on the right the first term disappears. Hence when $(x)$ is a null sphere with finite centre, and $(y)$ is a sphere of finite radius, our invariant $J$ is the ratio of the power of the centre of $(x)$ with regard to $(y)$ to its power with regard to the reflection of $(y)$ in the origin. When $(y)$ also becomes a null sphere $J$ is the ratio of the square of the distance of centres of $(x)$ and $(y)$ to the square of the distance of each from the reflection of the other.

This deduction is still at fault if $(x)$ be a null sphere, and $(y)$ a plane. Starting with the case where $(x)$ is a null sphere, and $(y)$ a sphere of finite radius, the power of the centre of $(x)$ with regard to $(y)$ divided by the radius of the latter is

$$
\frac{2(x y)}{\left(i x_{1}+x_{i}\right) y_{0}}
$$

The limit of this expression as $i y_{1}+y_{2} \doteq 0$ is the distance from the centre of $(x)$ to the plane $(y)$. Our invariant $J$ will thus give the distance from the centre of $(x)$ to the plane $(y)$ divided by its distance from the reflection of $(y)$ in the origin. The only cases where there is no geometrical interpretation for $J$ are where either

$$
x_{0}=i x_{1}+x_{2}=0 \quad \text { or } \quad y_{0}=i y_{1}+y_{2}=0
$$

Here one at least of the two spheres is a self perpendicular plane (or the plane at infinity) which does not make any particular angle with any thing, and is not at any particular distance from any point not in itself. 


\section{§3. Corresponding Expressions.}

$\Sigma$

Line.

Polarization in the Absolute.
$S$

Sphere.

Reflection in the origin.

Definition. The non-special linear complex in $\Sigma$ given by the equation

$$
x_{0}=0
$$

shall be called the notable complex. The line whose Klein coördinates are

$$
(0: i: 1: 0: 0: 0)
$$

shall be called the notable line. Notice that the notable line belongs to the notable complex.

Line of the notable complex.

Polar lines with regard to the notable complex.

Notable line.

Lines meeting notable line.

Lines of notable complex meeting notable line.

Product of tangents of distances of two lines, neither of which belongs to notable complex.

Product of tangents of distances of line belonging to notable complex but not intersecting notable line, and line neither belonging to complex nor meeting notable line.

Product of tangents of distances of two lines of notable complex neither of which meets notable line.

Product of tangents of distances of line meeting notable line but not belonging to notable complex and line belonging to complex but not neeting notable line.

These four correspondences are the most important established in the present paper.
Null sphere.

Spheres differing only in orientation.

Plane at infinity.

Planes.

Self-perpendicular planes.

Ratio of square of sine of half-angle of two not null spheres to square of sine of half angle which each makes with the reflection of the other in the origin.

Ratio of power of point with regard to a proper sphere, to its power with regard to the reflection of that sphere in the origin.

Ratio of square of distance of two points to square of distance of each from the reflection of the other in the origin.

Ratio of distance of point from plane to its distance from the reflection of plane in the origin. 
Intersecting lines.

Orthogonally intersecting lines.
Touching spheres.

Touching spheres, each of which touches the reflection of the other.

Thís last correspondence holds only in the general case. We leave to the reader the task of working out the special cases. Touching planes have the same orientation.

Pencil of lines not belonging to the Pencil of touching spheres. notable complex.

Pencil of lines of notable complex not meeting notable line.

Pencil of null spheres through an isotropic line.

It will be convenient to call an isotropic or self-perpendicular line an isotropic merely. The center of the null sphere on the right will lie on the isotropic. The infinite point of the isotropic will correspond to the line of the pencil that meets the notable line.

Pencil of lines of the notable complex which includes notable line.

Pencil of parallel self-perpendicular planes.

If we consider a point in $\Sigma$ every line through it intersects every line of the notable complex through that point, and the same is true of the lines in a plane.

Point and polar plane in null system Spheres containing isotropic. of notable complex.

It is more usual to say that a point in $\Sigma$ corresponds to an isotropic in $S$. If this mean that there is a one to one correspondence between them, well and good. But if it mean that the lines through a point are in one to one correspondence with the oriented spheres through an isotropic, it is certainly incorrect. Incidentally let us give the correspondence previously mentioned.

Asymptotic line of a surface.

Quadratic Regulus in notable complex whose lines do not meet notable one.

Conjugate regulus.

Conjugate reguli, not in notable complex.
Line of curvature of a surface.

Points of a circle.

Spheres through same circle.

Conjugate generations of a Dupin cyclide.

There are certain special lines worth remarking.

Left generator of Absolute.

Right generator of Absolute.

Left paratactic lines.

Right paratactic lines.
Sphere whose centre is origin.

Self-perpendicular plane through origin.

Spheres of equal radius and centres equidistant from origin.

Spheres whose centres are collinear with origin. 
Theorem 1).

If two not mutually absolute polar lines intersect orthogonally two other such lines, they will be paratactic and have an infinite number of common perpendiculars which are paratactic in the other sense. The latter will generate a regulus, whose conjugate consists of paratactic lines including the original two.
Theorem 1').

If two spheres, not the reflections of one another in the origin, touch two other such spheres, and their reflections, then either their radii are equal, and their centres equidistant from the origin, or they have their centres collinear with the origin. They will touch an infinite number of other spheres and their reflections in the origin. These will be one generation of an anchor ring whose center is the origin, the conjugate generation will include the original two spheres.

The theorem on the right is intuitively evident if we draw any sort of a picture, that on the left is one of the fundamental propositions in the theory of Clifford parallels. If two quadrics intersect in a pair of generators of one set, they will intersect in a pair of the other set also. If one be the Absolute, we have

Theorem 2).

If one set of generutors of a quadric be paratactic, the other set are paratactic in the opposite sense.
Theorem 2').

If one set of generating spheres of a Dupin cyclide have equal radii, and their centres equidistant from a fixed point, the other set have their centres collinear with this point and vice-versa. The cyclide is, under these circumstances, an anchor ring.

The surface on the left is named after the mathematician to whom we owe parataxy.

Clifford surface.

Theorem 3).

Through a given point and in a given plane there will be, in general, one left and one right paratactic to a given line.

Line tangent to Absolute not at a point of notable line.
Anchor ring whose axis passes through the origin.

Theorem $3^{\prime}$ ).

Through a given isotropic two spheres can, in general, be passed having their centres on a given line, and two of equal radius whose centres are equidistant from a given point. Sphere whose centre is at null distance from the origin. 
Pencil of tangents to Absolute not at point of notable line.

Line tangent to Absolute at point of notable line.

Pencil of such lines.

Point of Absolute and its polar in the notable complex, or plane tangent to Absolute and its pole in notable complex.

Parallel or pseudo-parallel lines.
Pencil of spheres through two isotropics which are in a self-perpendicular plane and reflections of each other in the origin.

Self-perpendicular plane.

Pencil of parallel self-perpendicular planes.

Isotropic whose plane with the origin is self-perpendicular.

Touching spheres one of whose common isotropics determines, with the origin, a self-perpendicular plane.

We get a good deal of light upon our correspondence by considering the corresponding transformation groups.

Group $g_{15} h_{15}$ of collineations and correlations.

Group $g_{6} h_{6}$, where $g_{6}$ includes all congruent transformations, and $h_{6}$ is product of $g_{6}$ and absolute polarization.

Group $g_{6}$ is composed of sub-group $\gamma_{6}$ which permutes the members of each set of generators of the absolute, and the assemblage $\eta_{6}$ which interchanges the two sets.

The group $\gamma_{6}$ is the product of two invariant subgroups $\gamma_{3} \gamma_{3}^{\prime}$. The former is the group of left translations, which keep all left generators of the absolute in place, the latter is the group of right translations.
Group $g_{15} h_{15}$ of contact sphere transformations.

Group $g_{6} h_{6}$ keeping invariant the assemblage of all spheres which either have their centres at the origin, or are self-perpendicular planes through the origin.

Group $g_{6}$ is composed of sub-group $\gamma_{6}$ which permutes the spheres whose centres are the origin, and the selfperpendicular planes through that point, and $\eta_{6}$ which interchanges the two systems.

The group $\gamma_{6}$ is the product of two invariant subgroups $\gamma_{3} \gamma_{3}^{\prime}$. The former is the group of all rotations about the origin, the latter carries each sphere into another whose centre is collinear with the given centre and the origin, and leaves each selfperpendicular plane through the origin in place. 


\section{§4. The Linear Complex.}

Suppose that we have in $\sum$ a linear complex,

$$
(a x)=0 \text {. }
$$

The corresponding complex in $S$ may be classified as follows : *

a)

$$
\sum_{i}^{1 \cdots 5} a_{i}^{2} \neq 0
$$

here we may write

$$
\frac{a_{1} x_{1}+a_{2} x_{2}+a_{3} x_{3}+a_{4} x_{4}+a_{5} x_{5}}{-i x_{0} \sqrt{\sum_{i}^{1} a_{i}^{5} a_{i}^{2}}}=\frac{a_{0}}{i \sqrt{\sum_{i}^{1 \ldots 5} a_{i}^{2}}} .
$$

This shows that the spheres of the complex cut the fundamental sphere (or plane) with coördinates

at the fixed angle

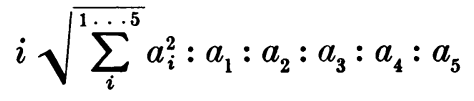

$$
\begin{gathered}
\cos ^{-1} \frac{a_{0}}{i \sqrt{\sum_{i}^{1 \cdots 5} a_{i}^{2}}} . \\
a_{0}=0 .
\end{gathered}
$$

The spheres are orthogonal to the fundamental sphere, which is null if $\sum_{i}^{1} \cdots 5 a_{i}^{2}=0$.

c) $\quad a_{0} \neq 0, \quad i a_{1}+a_{2} \neq 0, \quad \sum_{i}^{1} a_{i}^{5}=0$.

The ratio of the power of a fixed point to the radius of the sphere is a constant for all spheres of the complex. The fixed point may be taken as the centre of a fundamental null sphere.

$$
\text { d) } \quad a_{0} \neq 0, \quad i a_{1}+a_{2}=0, \quad \sum_{i}^{1} a_{i}^{5}=0 .
$$

Spheres are invariantly related to a certain self-perpendicular plane. It is not easy to give a description of this invariant relation.

e)

$$
a_{0}=a_{3}=a_{4}=a_{5}=0 \text {. }
$$

The complex of all oriented planes.

$$
a_{1}=a_{2}=a_{3}=a_{4}=a_{5}=0 .
$$

The complex of all null spheres.

* Conf. SNy DER, Über die linearen Complexe der Lies'chen Kugelgeometrie, Göttingen, 1895, and P. F. Sмiтh. On the Surfaces enveloped by Spheres belong into a linear Spherical Complex, Transactions of the American Mathematical Society, vol. 1 (1900). 
This complete classification is by means of invariants of the group of motions and inversions, and not by invariants of our group $g_{6} h_{6}$. Let us seek for another definition of the complex.

In our space $\sum$ let us take the non-special complex (29) and find the polar with regard to the corresponding null system of a line $(y)$. This will be

$$
\rho y_{i}^{\prime}=(a a) y_{i}-2(a y) a_{i} .
$$

When will the lines $(y)$ and $\left(y^{\prime}\right)$ also be mutually absolute polar? Clearly they must be polar with regard to the absolute polar complex

$$
(a \mid x)=0
$$

and so the directrices of the linear congruence determined by the two complexes.

Let $(r)$ be the coördinates of a line sought. Then if we write

$$
(a a)=h(a \mid a), \quad-1 \pm \sqrt{1-h^{2}}=l .
$$

$r_{0}: r_{1}: r_{2}: r_{3}: r_{4}: r_{5}=(h+l) a_{0}:(h+l) a_{1}:(h+l) a_{2}:(h-l) a_{3}:(h-l) a_{4}:(h-l) a_{5}$.

The two lines found from these equations shall be called the axes of the complex, the corresponding spheres, the axial spheres. Certain special cases must be taken up at once, as (31) will not always hold.

If the complex be self-polar with regard to the absolute, our method of finding the axes breaks down. We see, in fact

$$
a_{0}=a_{1}=a_{2}=0 \quad \text { or } \quad a_{3}=a_{4}=a_{5}=0 .
$$

In the former case, from (30) and the relation of the absolute polar lines,

$$
\begin{gathered}
y_{i}-\frac{2(a y) a_{i}}{(a a)}=-y_{i} \quad(i=3,4,5), \\
(a a) y_{i}=(a y) a_{i}, \quad y_{i}=a_{i} .
\end{gathered}
$$

We may take as an axis any line $\left(r_{0} r_{1} r_{2} a_{3} a_{4} a_{5}\right)$; in the latter case any line $\left(a_{0} a_{1} a_{2} r_{3} r_{4} r_{5}\right)$ in other words, any line of a definite paratactic system. If

$$
(a a)=0, \quad(a \mid a) \neq 0,
$$

the complex is special, and the axes are the line met by all lines of the complex, and its absolute polar. If

$$
(a a) \neq 0, \quad(a \mid a)=0, \quad 1 / h=0,
$$

the equations fo: the axis become

$$
r_{0}: r_{1}: r_{2}: r_{3}: r_{4}: r_{5}=a_{0}: a_{1}: a_{2}: i a_{3}: i a_{4}: i a_{5}
$$

the quantity $l / h$ is replaced by $i$. If

$$
(a a)=(a \mid a)=0 \text {, }
$$


the axes are $(a)$ and its absolute polar, two lines touching the absolute at the same point. If $h=1$, the axes fall together into a right generator of the Absolute. If $h=-1$, the axes fall together into a left generator of the absolute.

We shall mean by a general linear complex one which has a single definite pair of axes, not touching the absolute, nor lying therein. In such a case we may write

$$
\begin{aligned}
(r x) & =h(a x)+l(a \mid x), \\
(r \mid x) & =h(a \mid x)+l(a x), \\
(r \mid r) & =2 l[h(a a)-(a \mid a)], \\
\frac{(r x)}{(r \mid x)} & =\frac{l}{h} \text { if }(a x)=0 .
\end{aligned}
$$

Theorem 4).

$A$ general linear complex may be generated in two different ways by a line so moving that the product of the tangents of its distances from a fixed line is constant. The two fixed lines which may be so used are mutually absolute polar, and the two constants are reciprocals.*

Theorem $4^{\prime}$ ).

A general linear complex may be generated in two different ways by a sphere so moving that the sine of its half-angle with a given sphere bears a fixed ratio to the sine of its half angle with an equal sphere.

The general linear complex has an interesting sub-class.

General linear complex whose axes belong to the notable complex but do not intersect the notable line.
Complex of spheres with regard to which two fixed points have powers in a constant ratio.

The absolute invariant $l / h$ has, in $\Sigma$ a most interesting significance. Suppose that the axes are the two lines $\xi_{0}=\xi_{1}=0$ and $\xi_{2}=\xi_{3}=0$. Let us take an infinitesimal screw motion about them

$$
\begin{aligned}
& \xi_{0}^{\prime}=\xi_{0}+\xi_{1} d \theta, \\
& \xi_{1}^{\prime}=-\xi_{0} d \theta+\xi_{1}, \\
& \xi_{2}^{\prime}=\xi_{2}+\xi_{3} d \phi, \\
& \xi_{3}^{\prime}=-\xi_{2} d \phi+\xi_{3} .
\end{aligned}
$$

The expression $d \phi / d \theta$ will be the pitch of the screw with regard to $\xi_{0}=\xi_{1}=0$.

\footnotetext{
* A similar theorem will hold for the linear complex in euclidean space, see SEGEr loc. cit.
} 
If $(\eta)$ be a point of the absolute polar of the line $(\xi)\left(\xi^{\prime}\right)$

$$
\begin{gathered}
\sum_{i}^{0 \ldots 3} \xi_{i} \eta_{i}={ }^{0} \dddot{\Sigma}^{3} \xi_{i}^{\prime} \eta_{i}=0, \\
\left(\xi_{0} \eta_{1}-\xi_{1} \eta_{0}\right) d \theta+\left(\xi_{2} \eta_{3}-\xi_{3} \eta_{2}\right) d \phi=0 .
\end{gathered}
$$

A line through $(\xi)$ perpendicular to the direction of instantaneous motion will belong to the linear complex

$$
p_{01} d \theta+p_{23} d \phi=0, \quad(d \theta+d \dot{\phi}) x_{0}+i(d \theta-d \phi) x_{3}=0 .
$$

This axis of the complex has the coördinates

$$
(1: 0: 0:-i: 0: 0), \quad \frac{(r x)}{(r \mid x)}=-\frac{d \phi}{d \theta} .
$$

Theorem 5). In a general linear complex the product of the tangents of the distances of the lines from an axis is equal numerically to the pitch, with regard to that axis, of the instantaneous screv motion associated with the complex.

It will thus be convenient to define our invariant as the pitch of the complex. Let us revert to (32). These equations were independent of the relation $(x x) \equiv 0$.

$$
\begin{aligned}
(a r) & =h(a a)+l(a \mid a), \\
(a \mid r) & =h(a \mid a)+l(a a), \\
(r \mid r) & =2 l[h(a a)-(a \mid a)] .
\end{aligned}
$$

Now let $(y)$ and $\left(y^{\prime}\right)$ be two lines mutually polar with regard to the complex (i. e., polar with regard to the accompanying null system). We get from (30)

$$
\begin{aligned}
\left(r y^{\prime}\right) & =(a a)(r y)-2(a y)(a r) \\
& =-(a y)[2 l(a \mid a)+h(a a)]+l(a a)(a \mid y) \\
\left(r \mid y^{\prime}\right) & =(a a)(r \mid y)-2(a y)(r \mid r) \\
& =-(a y)[l(a a)+2 h(a \mid a)]+h(a a)(a \mid y) \\
\frac{(r y)}{(r \mid y)} & =\frac{h(a y)+l(a \mid y)}{l(a y)+h(a \mid y)} \\
\frac{\left(r y^{\prime}\right)}{\left(r \mid y^{\prime}\right)} & =\frac{[2 l(a \mid a)+h(a a)](a y)-l(a a)(a \mid y)}{[2 h(a \mid a)+l(a a)](a y)-h(a a)(a \mid y)}
\end{aligned}
$$

Eliminating $(a y) /(a \mid y)$, and remembering that

$$
(a a)=h(a \mid a), \quad h^{2}+l^{2}=-2 l,
$$


we have

$$
\frac{\left(r y^{\prime}\right)}{\left(r \mid y^{\prime}\right)} \cdot \frac{(r y)}{(r \mid y)}=\frac{l^{2}}{h^{2}}
$$

Let us also remember that two lines which are mutually polar with regard to a linear complex lie with the axes upon a quadratic regulus.

Theorem 6).

The polar of a line with regard to a general linear complex lies with it and the axes on a quadratic regulus, and the product of the tangents of the distances from an axis to two such polar lines is the square of the corresponding pitch of the complex.

\section{Theorem 6').}

The polar of a sphere with regard to a general linear complex lies with it and the axial spheres in one generation of a Dupin cyclide, and the product of the squared sines of the half angles which two such polar spheres make with one axial sphere divided by the corresponding product for the other axial sphere is the square of the pitch of the complex.

Unfortunately a line is not uniquely determined if we know a regulus whereon it lies, and the product of the tangents of its distances from a line of the regulus. Nevertheless, this theorem enables us quickly to determine the coördinates of the polar of a line with regard to a linear complex. If the axes be $(r)$ and $\left(r^{\prime}\right)$ any regulus containing them may be written

$$
\begin{gathered}
x_{i}=\rho^{2} r_{i}+2 \rho \sigma s_{i}+\sigma^{2} r_{i}^{\prime} . \\
(r r)=\left(r^{\prime} r^{\prime}\right)=(r s)=\left(r^{\prime} s\right)=(s s)-\left(r r^{\prime}\right)=0, \quad\left(r r^{\prime}\right)=(r \mid r) .
\end{gathered}
$$

If $(y)$ and $\left(y^{\prime}\right)$ be polar lines with regard to the complex

$$
\frac{\left(r y^{\prime}\right)}{\left(r \mid y^{\prime}\right)} \cdot \frac{(r y)}{(r \mid y)}=\frac{\sigma^{\prime 2}}{\rho^{\prime 2}} \cdot \frac{\sigma^{2}}{\rho^{2}}=\frac{l^{2}}{h^{2}}, \quad \frac{\sigma^{\prime}}{\rho^{\prime}} \cdot \frac{\sigma}{\rho}= \pm \frac{l}{h} .
$$

We must choose the upper sign, as we see by a continuous change from the case where $(y)$ and $\left(y^{\prime}\right)$ fall together. When all quantities and all lines here involved are real, the two polar lines will be separated in the regulus by the axes if $l / h<0$ and not separated if $l / h>0$.

Suppose that we have two linear complexes

$$
(a x)=0, \quad\left(a^{\prime} x\right)=0 .
$$

They have several absolute simultaneous invariants of which the simplest is

$$
\begin{gathered}
\frac{\left(a a^{\prime}\right)}{\left(a \mid a^{\prime}\right)}, \\
r_{0}=(h+l) a_{0}, \ldots, r_{3}=(h-l) a_{3},
\end{gathered}
$$




$$
\begin{aligned}
& r_{0}^{\prime}=\left(h^{\prime}+l^{\prime}\right) a_{0}^{\prime} \cdots r_{3}^{\prime}=\left(h^{\prime}-l^{\prime}\right) a_{3}^{\prime}, \\
&\left(r r^{\prime}\right)=\left(h h^{\prime}+l l^{\prime}\right)(a b)+\left(h l^{\prime}+l h^{\prime}\right)(a \mid b), \\
&\left(r \mid r^{\prime}\right)=\left(h h^{\prime}+l l^{\prime}\right)(a \mid b)+\left(h l^{\prime}+l h^{\prime}\right)(a b), \\
& \frac{\left(a a^{\prime}\right)}{\left(a \mid a^{\prime}\right)}= \frac{\left(r r^{\prime}\right)}{\left(\boldsymbol{r} \mid \boldsymbol{r}^{\prime}\right)}\left(h h^{\prime}+l l^{\prime}\right)-\left(h l^{\prime}+l h^{\prime}\right) \\
&\left(h h^{\prime}+l l^{\prime}\right)-\frac{\left(r r^{\prime}\right)}{\left(\boldsymbol{r} \mid \boldsymbol{r}^{\prime}\right)}\left(h l^{\prime}+l h^{\prime}\right)
\end{aligned}
$$

If we write

$$
\begin{gathered}
\frac{l}{h}=p, \quad \frac{l^{\prime}}{h^{\prime}}=p^{\prime}, \\
\frac{(a b)}{(a \mid b)}=\frac{\frac{\left(r r^{\prime}\right)}{\left(r \mid r^{\prime}\right)}\left(1+p p^{\prime}\right)-\left(p+p^{\prime}\right)}{\left(1+p p^{\prime}\right)-\frac{\left(r r^{\prime}\right)}{\left(r \mid r^{\prime}\right)}\left(p+p^{\prime}\right)} .
\end{gathered}
$$

Theorem 7)

Two general linear line complexes are in involution if the product of the tangents of the distances of two axes is equal to the sum of pitches divided by the product thereof plus one.

\section{Theorem 7')}

Two general linear sphere complexes are in involution if the square of the sine of the half angle formed by an axial sphere in each, divided by the square of the sine of the half angle of the other axial spheres, is equal to the sum of the corresponding pitches divided by the product thereof plus one.

Let us look briefly at the hitherto excluded case of the complex which is its own absolute polar. Here in $S$ we have $\infty^{2}$ axial spheres which either have the same radius and their centers at the same distance from the origin, or all have their centers on one line through the origin. In the former case the complex is. transformed into itself by any rotation about the origin, in the latter by any transformation of $\boldsymbol{\gamma}_{3}^{\prime}$. In the former case we shall have a pitch -1 . For if the axis will be $a_{0}: a_{1}: a_{2}: r_{3}: r_{4}: r_{5}$

$$
(r x)+(r \mid x)=2(a x)=0 .
$$

If $(x)$ be a line of the complex

$$
\frac{(r x)}{(r \mid x)}=-1
$$


The centre of a radical sphere will have the coördinates

$$
\frac{-r_{3}}{i a_{1}+a_{1}}, \quad \frac{-r_{4}}{i a_{1}+a_{1}}, \quad \frac{-r_{5}}{i a_{1}+a_{1}} .
$$

The square of its distance from the origin will be

$$
\frac{-\left(a_{0}^{2}+a_{1}^{2}+a_{2}^{2}\right)}{\left(i a_{0}+a_{1}\right)^{2}} \quad \text { since } \quad(r r)=0 .
$$

The radius of this sphere is

$$
\frac{i a_{0}}{\left(i a_{1}+a_{2}\right)} .
$$

The power of the origin with regard to this sphere is

$$
\frac{-\left(a_{1}^{2}+a_{2}^{2}\right)}{\left(i a_{1}+a_{2}\right)^{2}}
$$

and this is the square of the radius of the fundamental sphere.

Theorem 8). When the centre of the fundamental sphere is midway between the centres of the axial spheres, the former cuts the latter orthogonally, and the pitch of the complex is equal to -1 .

Suppose next that

$$
a_{0}=a_{1}=a_{2}=0 \text {. }
$$

The axial spheres will be any two spheres, symmetrical with regard to the origin,

$$
\begin{gathered}
r_{0}: r_{1}: r_{2}: a_{3}: a_{4}: a_{5}, \quad(r r)=0, \\
\frac{(r x)}{(r \mid x)}=1 .
\end{gathered}
$$

The radical plane of the two axial spheres is $0: 0: 0: a_{3}: a_{4}: a_{5}$.

Theorem 9). When the fundamental sphere is the radical plane of the axial spheres, the latter may be replaced by any other two spheres symmetrical with regard to the origin, and having the same line of centers, and the pitch of the complex equals 1 .

As a matter of fact we have a great deal more freedom in choosing our axial spheres in the present case, for, as the complex consists in the assemblage of all spheres orthogonal to the fundamental plane, it will be carried into itself by every translation parallel to this plane. We see, more generally, that when the spheres of the complex cut the fundamental sphere orthogonally they make equal angles with any two spheres mutually inverse with regard to the fundamental sphere.

Any linear complex which is not special may be carried into the complex $x_{0}=0$ by a transformation of the group $g_{15}$. 
Theorem 10).

The intersection of two linear complexes is a linear congruence whose directrices are mutually polar with regard to both.
Theorem 10').

The locus of points whose powers with regard to tuo equal spheres have a constant ratio is two spheres: differing only in orientation.

The theorem on the right is, of course, true when the two spheres are unequal in radius. It is interesting that there should be a connection between two such well known, but, apparently dissimilar theorems.

There are $\infty^{1}$ linear complexes which contain all the lines of a linear congruence.

Theorem 11).

If a linear congruence be given whose

directrices are not mutually absolute polar, we may in $\infty^{2}$ ways find two lines such that the product of the tangents of the distances from each to the lines of the congruence is constant.

Theorem 11').

If a system of spheres be given touching two spheres, we may in $\infty^{5}$ ways find two pairs of spheres symmetrical with regard to a fixed point so that the ratio of the sines of the halfangles made by the spheres of the system with the two spheres of each. pair is constant.

The increased freedom on the right is owing to our ability to choose the origin at will. Let us see if we can not make use of our freedom so that the two constants shall both be equal to 1 . Let the given complexes be

$$
(a x)=0, \quad(b x)=0 .
$$

The roots of the quadratic equation

$$
\lambda^{2}[(a c)+(a \mid a)]+2 \lambda \mu[(a b)+(a \mid b)]+\mu^{2}[(b b)+(b \mid b)]=0
$$

will, in general, be distinct and give two complexes with different axes.

Theorem 12).

If a linear congruence be given we may, in general, find two not mutually absolute polar lines such that the two distances from each to every line of the congruence are complementary.
Theorem 12').

If a system of spheres be given touching two spheres we may find two pairs of spheres symmetrical with regard to any point in general position, so that the spheres of the system make equal angles with the two. spheres of each pair. 
On the right a point " in general position" means one not on the locus that would give the quadratic equation (35) equal roots.

If we have a quadratic regulus given by

$$
(a x)=(b x)=(c x)=0,
$$

we may in $\infty^{3}$ ways find three sets of roots for the equation

$$
\begin{aligned}
& \lambda^{2}[(a a)+(a \mid a)]+\mu^{2}[(b b)+(b \mid b)]+\nu^{2}[(c c)+(c \mid 0)] \\
& \quad+2 \mu \nu[(b c)+(b \mid c)]+2 \nu \lambda[(c a)+(c \mid a)]+2 \lambda \mu[(a b)+(a \mid b)]=0 .
\end{aligned}
$$

Theorem 13).

$A$ regulus may be generated in $\infty^{3}$ ways by lines whose two distances from each of three fixed lines, no two of which are mutually absolute polar, are complementary.
Theorem 13').

A generation of a Dupin cyclide may be formed in $\infty^{6}$ ways by spheres: cutting at equal angles the two spheres of each of three pairs symmetrical with regard to a fixed point.

\section{§5. The Dual Projective Geometry.}

Let us return to our fundamental equations (31) which may be rewritten

$$
\frac{h-l}{h+l}=\frac{(a a)+(a \mid a) \mp V \overline{(a \mid a)^{2}-(a a)^{2}}}{(a a)-(a \mid a) \pm \sqrt{(a \mid a)^{2}-(a a)^{2}}}= \pm \frac{\sqrt{(a \mid a)+(a a)}}{\sqrt{(a \mid a)-(a a)}}
$$

If, now, we leave $a_{0}: a_{1}: a_{2}$ invariant, but change $a_{3}: a_{4}: a_{5}$ into $\rho a_{3}: \rho a_{4}: \rho a_{5}$, we see that the two axes $(r)$ are unaltered. We have thus $\infty^{1}$ complexes which have the same axes or axial spheres, and are said to be coaxal. We may thus conceive the two axes or axial spheres as a new space element, determined by two triads of separately homogeneous coördinates.

Theorems 14 and 14'). There is a one to one correspondence between the pairs of points of two planes (projectively defined), the pairs of lines mutually polar with regard to a quadric and the pairs of spheres symmetrical with regard to a fixed point.

The system of geometry with any one of these as element may be called dual projective. The case where the element is the pair of axes of a coaxal system of 
complexes has already been treated at length.* Let us therefore confine ourselves to the relation between the geometry of point-pairs in two projective planes, and pairs of spheres symmetric with regard to a fixed point.

We must begin by taking account of the lapses in the one to one correspondence described in theorem 14. Let us call the plane $a_{0}: a_{1}: a_{2}$ the left plane while $a_{3}: a_{4}: a_{5}$ is the right plane. The conics

$$
a_{0}^{2}+a_{1}^{2}+a_{2}^{2}=0, \quad a_{3}^{2}+a_{4}^{2}+a_{5}^{2}=0
$$

shall be called the fundamental conics. Suppose, first, that the spheres coalesce into a self-perpendicular plane through the origin

$$
r_{0}=r_{1}=r_{2}=(r r)=0 \text {. }
$$

To this will correspond every pair of points

$$
\left(a_{0} a_{1} a_{2}\right), \quad\left(r_{3} r_{4} r_{5}\right), \quad a_{0}^{2}+a_{1}^{2}+a_{2}^{2} \neq 0 .
$$

We see thus, that to a self-perpendicular plane through the origin will correspond any point in the left plane, not on the fundamental conic, and a definite point of the fundamental conic in the right plane. In the same way, to a sphere whose center is the origin will correspond a definite point of the fundamental conic in the left plane, and any point not on the fundamental conic in the right.

Suppose, thirdly, that we have

$$
r_{0}^{2}+r_{1}^{2}+r_{2}^{2}=r_{3}^{2}+r_{4}^{2}+r_{5}^{2}=0
$$

Every complex of the coaxal system is special, and the spheres form a pencil of tangent spheres through two isotropics, the reflections of each other in the origin, their plane being self perpendicular. Such a pencil will correspond to each pair of points on the two fundamental conics. Conversely if the point in neither plane lie on the fundamental conic, we have a single pair of spheres. We have also a single pair of coincident spheres when one point lies on a fundamental conic and the other does not. When both points lie on the fundamental conics

* The euclidean case was trented first, and at great length, by STUDY in the third part of his Geometrie der Dynamen, Leipzig, 1903. The same writer sketched out the non-euclidean cases without proofs in an article Zur Nichteuklidischen und Liniengeometrie, Jahresbericht der Deutschen Mathematikervereinigung, vol. 11 (1902). The elliptic case was then studied in detail by the author in his Dual Projective Geometry of Elliptic and Spherical Space, Greifswald, 1904, and the hyperbolic by Веск, Die Strahlenketten im hyperbolischen Raume, Hannover, 1905. For a briefer account of the non-euclidean cases see the author's Non-Euclidean Geometry, already cited, Ch. X. 
we have a pencil of spheres through two parallel isotropics the reflections of each other in the origin and in a self-perpendicular plane through the origin.

Before discussing figures generated by sphere-pairs, let us notice that our dual coördinates $\left(a_{0} a_{1} a_{2}\right)\left(a_{3} a_{4} a_{5}\right)$ are capable of another interpretation besides that of the system of coaxal complexes. It is, in fact, immediately evident that

$$
\frac{a_{3}}{\sqrt{a_{3}^{2}+a_{4}^{2}+a_{5}^{2}}}, \frac{a_{4}}{\sqrt{a_{3}^{2}+a_{4}^{2}+a_{5}^{2}}}, \frac{a_{5}}{\sqrt{a_{3}^{2} a_{4}^{2}++a_{5}^{2}}}
$$

are the direction cosines of the line of centers of the two spheres. On the other hand if $\theta_{1}$ and $\theta_{2}$ be the angles which the two spheres make with the spheres $(i 10000),(i 01000)$ whose common center is the origin,

$$
a_{0}: a_{1}: a_{2}=1: i \cos \theta_{1}: i \cos \theta_{2} \text {. }
$$

The fundamental relation in dual sphere geometry is touching. If

$$
u_{0} a_{0}+u_{1} a_{1}+u_{2} a_{2}=0, \quad v_{3} a_{3}+v_{4} a_{4}+v_{5} a_{5}=0
$$

the two spheres $\left(a_{0} a_{1} a_{2}\right)\left(a_{3} a_{4} a_{5}\right)$ touch the two spheres $(u)(v)$.

Suppose that we have a fixed point in one plane, and the points of a line ir the other. We have $\infty^{1}$ spheres touching a pair of axial spheres, and with their centres either on a line through the origin, or on a sphere whose center is the origin, and with equal radii.

\section{Representing Planes.}

Point in one plane not on fundamental conic and line in the other not tangent to the fundamental conic.

Polar and pole with regard to fundamental conics of foregoing point and line.
S.

One generation of anchor ring whose centre is the origin.

Other generation of same ring.

The next most interesting one-parameter family is given by two projective ranges in the two planes

$$
a_{i}=\lambda b_{i}+\mu c_{i}, \quad i=0,1,2,3,4,5 .
$$

There are three sub-cases.

a) The points of the fundamental conics correspond. We may imagine that these are $(b)$ and $(c)$. Let $(r)$ and $\left(r^{\prime}\right)$ be any two spheres of the system. 


$$
\begin{aligned}
(a a) & =2 \lambda \mu(b c), \quad\left(a^{\prime} a^{\prime}\right)=2 \lambda^{\prime} \mu^{\prime}(b c), \\
(a \mid a) & =2 \lambda \mu(b \mid c), \quad\left(a^{\prime} \mid a^{\prime}\right)=2 \lambda^{\prime} \mu^{\prime}(b \mid c), \\
h & =\frac{(b c)}{(b \mid c)}=h^{\prime}, \quad l=l^{\prime}, \\
\left(r r^{\prime}\right) & =\left(h h^{\prime}+l l^{\prime}\right)\left(a a^{\prime}\right)+\left(h l^{\prime}+l h^{\prime}\right)\left(a \mid a^{\prime}\right), \\
& =\left(h^{2}+l^{2}\right)\left(\lambda \mu^{\prime}+\lambda^{\prime} \mu\right)(b c)+2 h l\left(\lambda \mu^{\prime}+\lambda^{\prime} \mu\right)(b \mid c), \\
& =0, \quad \text { since } h^{2}+l^{2}=-2 l .
\end{aligned}
$$

Projective ranges where the points of $\mid$ Pencil of mutually touching spheres fundamental conics correspond. $\quad$ which touch two axial spheres.

b) One intersection with a fundamental conic corresponds to one such in the other plane. This is an unsymmetrical case which we pass over.

c) The intersections with the fundamental conics do not correspond.

The one parameter family of spheres all touch a pair of axial spheres and generate a surface which we shall call a $C$ surface. Let us find its order. If

$$
\begin{aligned}
\lambda / \mu & =z, \\
(a a) & =z^{2}(b b)+2 z(b c)+(c c), \\
(a \mid a) & =z^{2}(b \mid b)+2 z(b \mid c)+(c \mid c), \\
r_{0} & =v^{\prime} \overline{[(b b)-(b \mid b)] z^{2}+2[(b c)-(b \mid c)] z+[(c c)-(c \mid c)]}\left(z b_{0}+c_{0}\right), \\
r_{3} & =\sqrt{[(b b)+(b \mid b)] z^{2}+2[(b c)+(b \mid c)] z+[(c c)+(c \mid c)]}\left(z b_{3}+c_{3}\right), \\
r_{0} & =\sqrt{f_{2}(z)}\left(z b_{0}+c_{0}\right), \quad \alpha_{3}=\sqrt{\phi_{2}(z)}\left(z b_{4}+c_{3}\right) .
\end{aligned}
$$

If $0: x_{1}: x_{2}: x_{3}: x_{4}: x_{5}$ be a point of this sphere

$$
\sqrt{f_{2}(z)} \sum_{i}^{1} \dddot{2}^{2}\left(z b_{i}+c_{i}\right) x_{i}+{\overline{\phi_{2}(z)}}^{3} \dddot{\sum}_{i}^{5}\left(z b_{i}+c_{i}\right) x_{i}=0 .
$$

To find the envelop of these spheres we must square this expression getting an expression of the fourth order in $z$, quadratic in $x_{i}$, differentiate with respect to $z$ and eliminate that parameter. This gives an expression of the fourteenth order in $x_{i}$.

Theorem 15'. The surfaces $C$ are of the twenty-eighth order, with the circle at infinity as a curve of the fourteenth order. 
This theorem would be of uncommonly little importance, were it not for a very general and remarkable property of $C$ surfaces. Suppose that we have any analytic congruence of spheres

$$
b_{i}=b_{i}(u v)
$$

Let us find the sphere which touches $(b)$, its adjacent sphere

$$
(b)+\frac{\partial(b)}{\partial u} d u+\frac{\partial(b)}{\partial v} d v
$$

and the reflections of the two in the origin,

$$
\begin{gathered}
a_{0} b_{0}+a_{1} b_{1}+a_{2} b_{2}=a_{3} b_{3}+a_{4} b_{4}+a_{5} b_{5}=0 \\
\sum_{i}^{0 \ldots 2} a_{i}\left(\frac{\partial b_{i}}{\partial u} d u+\frac{\partial b_{i}}{\partial v} d v\right)=\sum_{i}^{3 \ldots 5} a_{i}\left(\frac{\partial b_{i}}{\partial u} d u+\frac{\partial b_{i}}{\partial v} d v\right)=0
\end{gathered}
$$

Let us assume that

$$
\left|a_{0} \frac{\partial b_{1}}{\partial u} \frac{\partial b_{2}}{\partial v}\right| \neq 0, \quad\left|a_{3} \frac{\partial b_{4}}{\partial u} \frac{\partial b_{5}}{\partial v}\right| \neq 0 .
$$

We choose such a $(b)$ (said to be in the general position) that neither of these determinants vanishes. $\mathrm{W}^{\top}$ e may then write

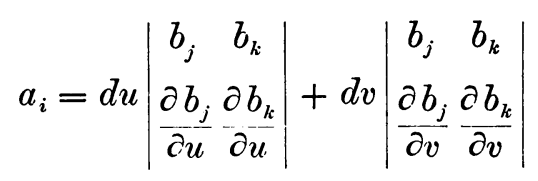

where $i, j, k$ are circular perınutations of $0,1,2$ and $3,4,5$. Varying $d u$ and $d v$ at will, we have

Theorem 16'. If an analytic congruence of spheres be given and a point chosen which is neither the vertex of a cone which is the locus of their centres, nor the centre of $\infty^{1}$ spheres each passing through the centres of $\infty^{1}$ spheres of the congruence of equal radius, then if a sphere of the congruence be chosen, the spheres which touch it and the adjacent spheres of the congruence, as well as the reflections in the fixed point, will generate a $C$ surface.*

An interesting two parameter family is the chain congruence, given by

$$
a_{i}=\lambda b_{i}+\mu c_{i}+\nu d_{i}, \quad i=0,1,2, \quad \text { or } \quad 3,4,5
$$

* This is, of course, the line-sphere transform of the non-euclidean form of Hamilton's famous theorem on the cylindroid. See his paper on Systems of Rays, Transactions of the Royal Irish Academy, vol. 15 (1829), and the author's Non-euclidean Gcometry, chap. X. 
We pass over the discussion of the various possible special cases, and take the general case where we can solve three of the equations for $\lambda, \mu, \nu$ and substitute in the other three. We may here write

$$
a_{0}=u_{0} a_{3}, \quad a_{1}=u_{1} a_{4}, \quad a_{2}=u_{2} a_{5} .
$$

The spheres which touch two spheres of the congruence and their reflections in the origin will generate the conjugate congruence

$$
b_{3}=u_{0} b_{0}, \quad b_{4}=u_{1} b_{1}, \quad b_{5}=u_{2} b_{2} .
$$

General projectivity between the two Chain congruence. planes.

Contragredient projectivity.

Conjugate congruence.

When a projective relation is established between two planes, one is thereby established between the ranges on any two corresponding lines in them.

Theorem 17'). The spheres of a chain congruence which touch one of the conjugate congruence and its reflection in the origin generate a $C$ surface.

Are there any spheres of our congruence which touch one of the conjugate congruence without touching its reflection? Let us write

$$
\begin{aligned}
& r_{0}=a_{0}, \quad r_{3}=\frac{h-l}{h+l} a_{3}, \quad s_{0}=b_{0}, \quad s_{3}=\frac{h^{\prime}-l^{\prime}}{h^{\prime}+l^{\prime}} b_{3}, \\
& \text { If }(r s)=0, \\
& \quad\left(a_{0} b_{0}+a_{1} b_{1}+a_{2} b_{2}\right)\left[1+\frac{(h-l)\left(h^{\prime}-l^{\prime}\right)}{(h+l)\left(h^{\prime}+l^{\prime}\right)}\right]=0 .
\end{aligned}
$$

If the first factor vanish $(a)$ touches $(b)$ and also its reflection. If the second vanish

$$
h h^{\prime}+l l^{\prime}=0 .
$$

But there will be a singly infinite set of values for $(a)$ and $(b)$ in the two congruences satisfying that equation. Hence $\infty^{1}$ spheres of each congruence touch $\infty^{1}$ of the other, but not their reflections.

Theorem 18). The spheres of a chain congruence which touch a sphere of the conjugate but not its reflection in the origin form one generation of a Dupin cyclide, the other generation belonging to the conjugate congruence.

Theorem 19). The spheres which touch pairs of spheres of one generation of a Dupin cyclide and their reflections in a fixed point generate a chain congruence.

The chain congruence has many other beautiful properties, easily proved from the corresponding properties of the chain congruences of lines by means of the line-sphere transformation. 
In closing one remark of a general character may not be out of place. The method of polar reciprocation in a quadric, descriptively considered, is a means of illustrating the principle of duality. Metrically, it shows the perfect correspondence of the concepts of distance and angle in non-euclidean space. The line-sphere transformation, descriptively considered, is one of the most beautiful examples under the general type of contact transformation. Metrically, as we have seen, it brings out numerous relations between the distances of lines and the angles of spheres. Is it not probable that there exists a large class of contact transformations which are not merely descriptive in character, but may be used to establish interesting metrical relations among elements of various sorts? Can we, without a total loss of naturalness, include all contact transformations in such a class?

Cambridge, Mass., July, 1910. 